\title{
A Crowd Modeling Framework for Socially Plausible Animation Behaviors
}

\author{
Seung In Park, Chao Peng, Francis Quek, Yong Cao \\ Department of Computer Science, Virginia Tech, USA \\ \{spark80, chaopeng, quek, yongcao\}@vt.edu
}

\begin{abstract}
This paper presents a framework for crowd modeling that produces socially plausible animation behaviors. Our high-level behavioral model is able to produce appropriate animated behavior that includes synchronized body-orientation and gesture of individual actors within the simulation. Because the model operationalizes a well-founded social-linguistic Common Ground (CG) theory of human interaction, the behavior chains form meaningful interactions among the actors. The model includes micro-behaviors relating to CG theory, and macro-behavior relating to the animation context. This allows reuse of the micro-behaviors as animation contexts change and flexible adaptation to different animation contexts.
\end{abstract}

Keywords: Crowd simulation, behavior control, character animation

\section{Introduction}

Producing realistic simulations of multiple characters is a challenging task. To construct plausible facsimiles of real crowd movements, it is not enough to provide animated characters merely with visually pleasing motion styles and variety of appearance in a simulated crowd. Providing a higher behavioral fidelity plays a vital role in the simulation. When multiple characters are involved in a simulation, realism is enhanced when the actors interact and coordinate behaviors with other characters in a socially plausible human-like manner.

In our previous work $[22,23]$, we presented a group coordination model informed by Common Ground (CG) theory by Clark [5] to incorporate the impact of social interaction among group members in crowd simulations. Given that a crowd typically consists of many number of small groups and crowd dynamics emerges from the interaction of the groups $[6,12]$, we proposed a coordination model reflecting the communication process of group members by which a cohesive movement is maintained. A set of experiments showed notable differences resulted in the simulations in terms of congestion level and crowd circulation pattern when our coordination model is enabled. In this paper, we extend the coordination model to accommodate high-level socially-aware behavioral realism of animated characters in a crowd simulation.

A key contribution of this paper is that we present a framework for crowd modeling that produces socially plausible animation behaviors, where individuals within the crowd belong to different social groups, and where the behavior 
responds to events and scenarios in the simulation environment. The high-level behavioral model is able to produce appropriate animated behavior that includes synchronized body-orientation and gesture of individual actors within the simulation. Because the model operationalizes a well-founded social-linguistic CG theory of human interaction, the behavior chains form meaningful interactions among the actors. The model includes micro-behaviors relating to CG theory, and macro-behavior relating to the animation context (e.g., military coordination strategies, cultural preferences, etc.). This allows 'reuse' of the microcoordination behaviors as animation contexts change and flexible adaptation to different animation contexts.

The remainder of this paper is organized as follows: Section 2 provides a brief overview of the related work in crowd simulation. We describe our framework for crowd modeling in Section 3. Section 4 presents our implementation choices and the results of simulations. We draw conclusions and provide possible future research directions in Section 5.

\section{Related Work}

In the past years a great deal of effort has been put in the field of group and crowd simulation. These include approaches to variants of agent-based methods incorporated with local rules [20,28], social force models [9], velocity models [1, $8]$, and physics based approaches $[11,21]$. Recently much attention has been given to model human-like behaviors and autonomous agents. These include incorporating psychological effects $[10,25]$, culture-specific behavior patterns $[7$, $32]$, and human cognition process $[14,29,34]$ into a crowd model. To imitate real crowd dynamics, some studies focus on creating realistic human walking paths [3,27], leader-following behaviors [16], and spatial group structure [19]. However these studies place much emphasis on collision avoidance and path planning, and animated characters are mostly equipped with simple ambulatory action skills.

There are a number of studies on embodied conversational agents (ECAs), which aim to model realistic non-verbal conversational behaviors of animated characters $[4,13,24,30]$. ECAs combine synthesized speech with hand gestures, head movements and facial expressions to create believable social avatars in conversational setting. Most of these works do not address dynamic movement and positioning of characters, hence restrict the scope of applications.

Numerous approaches to motion synthesis and editing have been proposed to provide physically realistic human motions $[17,18]$. To simulate sophisticated character motions, character behavior adaptation techniques accounting for the environmental objects and other characters have been proposed in $[15,27,31$, 33]. The main limitation of these approaches is that only reactive actions are generated with local information about the surroundings. Some approaches concern spatial and temporal alignments of gestures among characters, however the motion selection is not tightly coupled to the underlying simulation model. Therefore the sequence of character gestures does not form socially meaningful 
interactions. In our framework, behaviors for a character are determined by the dynamic social and environmental context in which the character functions.

\section{Crowd Model Framework}

A theory of CG by Herbert Clark from social-psychology and linguistics [5] supports the theoretical foundation of our group coordination model. CG refers to the mutual understanding accumulated among participants in a joint activity. It is achieved through an interactive process in which participants exchange evidence in order to reach mutual understanding. We model the process of performing group navigation in the CG framework, in which members of a group must communicate and maintain a state of mutual understanding for the successful joint navigation.

We use a scenario, which will be referenced throughout this section, to describe how the joint navigation is framed in the CG model and how the behavior is consistently carried through from simulation through to character animation. Suppose that $A$ and $B$ walk in a shopping mall. $A$ spies a bag in a store that she wants to look at, and informs $B$ of her plan to visit the store and to return to their current location, $x$. We denote the plan to split up and reunite at $x$ as $\mathcal{M}$. For the plan to succeed, $A$ needs to know that $B$ knows the plan $\mathcal{M}$. This, however, is insufficient for coordination. $B$ needs to know that $A$ knows that he is privy to $P$, otherwise he might not be convinced that $A$ will return to $x$. Furthermore, if the agreement ends here, $A$ may not know that $B$ knows that she knows the plan, and may, therefore not be confident to execute the plan. Hence, $A$ needs to know that B knows that she knows the plan.

The CG may be arrived at verbally, or may be enacted through action. For example, $A$ may signal her intention by pointing toward the store and pointing to their current location $x$. This requires that $B$ be within the range of sight and be looking at $A$. $A$ needs to see that $B$ is looking at her, and has signaled agreement (e.g., by nodding). $B$ needs to see that $A$ sees his nodding. Finally $A$ needs to see that $B$ sees that she has seen and acknowledged the plan. As described here, a behavior chain of actors is generated over the course of communication at simulation time, and this information can be associated with character animation. We define two kinds of behaviors; micro-behavior and macro-behavior. Micro-behavior relates to the communicative actions and gestures towards mutual understanding among group members, and macro-behavior refers to overall actions determining the spatial movements of members to accomplish a joint navigation. $A$ 's proceeding to a store and returning to $x$, and $B$ 's waiting at $x$ are macro-behaviors in our scenario.

\subsection{System Overview}

Figure 1 shows an architecture of our crowd modeling framework, composed of simulation engine and rendering engine. A simulation engine includes a goal generator, situation assessment module and coordination planner. In our simulation 


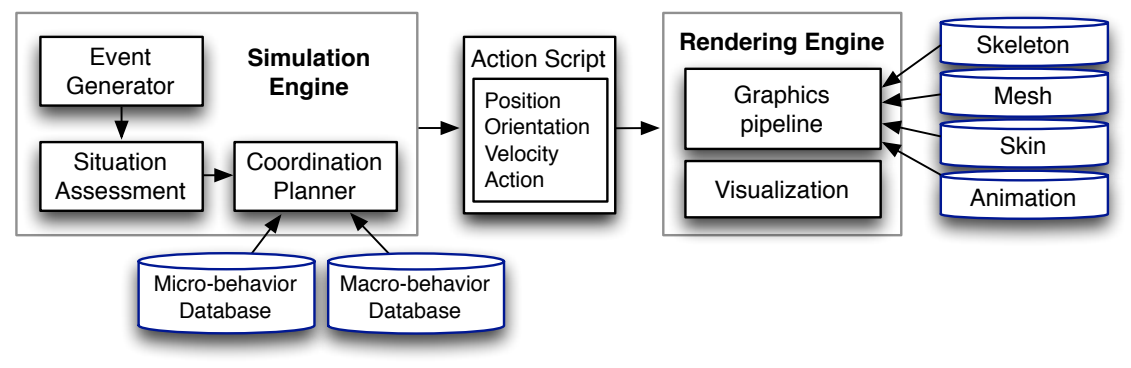

Fig. 1. System architecture

each character is motivated by stochastically generated and/or environmentally driven goals. These goals are represented as specific and definable geographic points in a given virtual environment. A random event generator triggers characters to have these goals. For example, a members of groups may have to visit the restroom (stochastically generated goal), or a member of a group may see a store that sells something she is interested in (environmentally driven goal).

When a character receives a goal event trigger, a situation assessment module evaluates the spatial relation among group members and the state of immediate environment for the character. A coordination planner collects the result of situation evaluation and selects appropriate micro- and macro-behaviors from databases in which each of micro- and macro behaviors are coded separately as alternatives. As a result, information of position, orientation, velocity, and action specification defining kind of behavior and activation time is generated for each character at each frame. The information forms an action script and is transmitted to a rendering engine. A rendering engine works in accordance with databases of characters' skeleton, mesh, skin, and pre-canned animation cycles to render out the animated actors given the input of action script. The set of animation sequences has been designed to support the repertoire of motions defined in the micro- and macro-behavior databases.

\subsection{Coordination Model}

Given an event goal trigger, a character initiates communication with group members by exhibiting a micro-behavior. As a response to the initiator's action, the rest of group members present proper micro-behaviors. Table 1 shows a general flow of the interactive process to establish CG between any two members $A_{i}$ and $A_{j}$ of group $\mathcal{G}_{k}$. A communicative action taken at each step is a microbehavior, and a particular set of macro-behaviors is adopted as a consequence of the coordination.

Micro-behavior We show examples of the high-level communication intents and how they are represented as bodily behaviors in Table 2. Available micro- 
Table 1. Interactive process to establish CG

\begin{tabular}{ll}
\hline step & Action Description \\
\hline 1 & $A_{i}$ acts to satisfy conditions for communication initiation. \\
2 & $A_{i}$ requests attention by performing a signaling action. \\
3 & $A_{j}$ gives attention to $A_{i}$. \\
4 & $A_{i}$ proposes a macro-behavior plan, $\mathcal{M}_{\beta}$ (i.e. select $\mathcal{M}_{\beta}$ ). \\
5 & $A_{j}$ signifies acknowledgement for $\mathcal{M}_{\beta}$. \\
6 & $A_{i}$ comfirms $A_{j}$ 's acknowledgement. \\
\hline 7 & If CG is achieved, \\
& Return TRUE (i.e. execute $\mathcal{M}_{\beta}$ ), \\
& Else Return FALSE (coordination failed).
\end{tabular}

behaviors are not limited to the given list, and any action reflecting what human carries out during the course of interaction can be added.

Table 2. Examples of communication intents and corresponding micro-behaviors

\begin{tabular}{ll}
\hline Communication Intent & Micro-behavior \\
\hline Initiate communication & Call out, move toward, turn toward \\
Request attention & Call out, look at other, wave hands \\
Give acknowledgement & Look at other, head nod \\
Suggest a macro-behavior & Point toward direction \\
\hline
\end{tabular}

A specific micro-behavior is selected by the result of situation assessment. Figure 2(a) shows a sensory model of a character, by which the information on the surroundings situation is collected. Touch is sensed within range of character radius $r$, hearing can be omnidirectional with range limitation $d_{h}$, and vision is directional and is effective up to a range, $d_{v}$, along its body direction (for simplicity, body orientation is synonymous to gaze direction in our model) and within a field of view defined by an angle, $\alpha$. Details on the situation assessment process may be found in [22].

Figure 2(b) illustrates possible spatial relations of any two agents, $A$ and $B$. In this case, $A$ is the initiator of an interactive exchange. Hence $A$ has to evaluate the state of perception of $B$ depending on where she is with respect to $B$. There are six possible spatial relations, labeled $A_{1} \ldots A_{6}$ in the figure. If $A$ determines it is in the $A_{3}$ position, it is outside the immediate perception of $B$ and has to move into a position where one of her means of communication is possible. A micro-behavior may then be selected to move within $B$ 's field of view (for example, position $x_{i}$ ), and wave at $B$ to get his attention. An alternative microbehavior choice may be to have $A$ walk into hearing range (e.g., position $x_{j}$ ), and calling out to $B$ to get his attention. If the former micro-behavior is selected, $B$ 


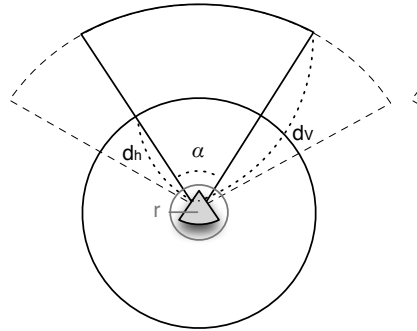

(a)

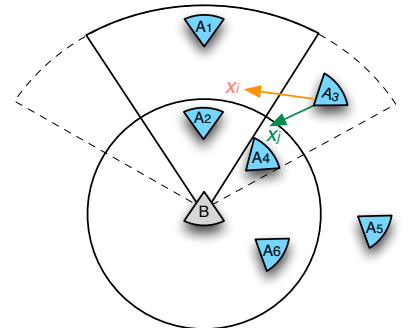

(b)

Fig. 2. (a) Sensory model of a character (b) Possible spatial relations of group members

may respond by looking at $A$ and nodding his head in acknowledgement. Then $A$ may suggest a macro-behavior plan $\mathcal{M}$ by pointing toward the store and then to the ground. $B$ will have to look at where $A$ is pointing and turn back to $A$ to signal that he has seen what she is pointing at and acknowledges her plan by nodding. Finally $A$ has to show that she understands that $B$ is in on the plan by nodding back. This range of possible choices picked either randomly or based on some actor-specific preference may serve to make the simulation seem less mechanistic or fully deterministic, thereby adding to realism. The key is that the design of the micro-behaviors conform to the rules for CG negotiation outlined at the beginning of this section. Similarly, each configuration $A_{1}, \ldots, A_{6}$ can initiate a set of appropriate micro-behaviors.

Spatial movement parameters (i.e., desired body orientation and position) in micro-behaviors are derived by considering goal locations, character positions, and orientations. Given the length of animated motion, we empirically determine an appropriate synchronized time lag between a character's micro-behavior and the response of the others. The time lag for walking and turning motions are adjusted by considering the distance to travel and angle to rotate, respectively. Table 3 shows a resulting action script specifying a sequence of micro-behaviors for $A$ and $B$ in the example just cited. This action script is applicable to the scenario we described in the beginning of this section. $x_{a}$ and $x_{b}$ are positions of $A$ and $B$ respectively, $x_{i}$ is some point within $B$ 's field of view, $x_{s}$ is a store location, and $x_{s}^{\prime}$ is where $A$ is pointing at. $t_{0}$ is a start time of the interaction. A length of an animated motion is denoted as $f_{\text {motion_name }}^{\text {actor }}$, in which motion_name represents a kind of motion, and actor_id is an operator of the motion.

Macro-behavior Given the ability to signal to other group members to adapt their actions, actors select and perform a macro-behavior plan (macro-plan) to accomplish the joint navigation. A macro-plan consists of a series of macrobehaviors and is completed by the actors involved, each doing their participatory actions in particular roles. The specific kinds of actions in macro-plan is determined by the domain of simulation. For instance, in a military scenario, a set of 
Table 3. An example of micro-behavior action script for $A$ and $B, A_{3}$ condition

\begin{tabular}{|c|c|c|c|c|}
\hline \multirow{2}{*}{ CG step } & \multicolumn{4}{|c|}{ Micro-behavior and time stamp } \\
\hline & \multicolumn{2}{|c|}{$A$, motion and activation time } & \multicolumn{2}{|c|}{$B$, motion and activation time } \\
\hline 1 & Walk to $x_{i}$ & $t_{0}$ & & \\
\hline 2 & Wave at $x_{b}$ & $t_{1}: t_{0}+f_{\text {walk_to_- } x_{i}}^{A}$ & & \\
\hline 3 & & & Turn towards $x_{a}$ & $t_{2}: t_{1}+f_{\text {wave }}^{A}$ \\
\hline $4 \mathrm{a}$ & Point toward $x_{s}$ & $t_{3}: t_{2}+f_{\text {turn }}^{B}$ & & \\
\hline $5 \mathrm{a}$ & & & Turn towards $x_{s}$ and nod & $t_{4}: t_{3}+f_{\text {point }}^{A}$ \\
\hline $4 \mathrm{~b}$ & Point down & $t_{5}: t_{4}+f_{\text {turn }^{\prime}}^{B}+f_{\text {nod }}^{B}$ & Nod head & $t_{6}: t_{5}+f^{A}$ \\
\hline 6 & Nod head & $t_{7}: t_{6}+f_{\text {nod }^{\prime}}^{B}$ & & $\int_{6} \cdot \iota_{5} \top J_{\text {point }}^{\prime}$ \\
\hline
\end{tabular}

macro-behaviors may be to send out a reconnaissance and wait, to divide and proceed to different goals, or to spread out and head to original goal position.

Table 4. A list of MacroCSs in our simulation

\begin{tabular}{|c|c|}
\hline Macro-plan & Action Description \\
\hline \multirow[t]{2}{*}{$\mathcal{M}_{1}:$ divide-and-proceed } & $\begin{array}{l}A_{i} \text { proceeds to a goal } x_{p} \\
\text { If the goal is accomplished, } \\
\text { proceed to a next goal } x_{q}\end{array}$ \\
\hline & $A_{j}$ proceeds to a final destination $x_{r}$ \\
\hline \multirow[t]{2}{*}{$\mathcal{M}_{2}$ : divide-and-wait } & $\begin{array}{l}A_{i} \text { proceeds to a goal } x_{p} \\
\text { If the goal is accomplished, } \\
A_{j} \text { returns to its previous location }\end{array}$ \\
\hline & $\begin{array}{l}A_{j} \text { stays and waits until } A_{i} \text { returns } \\
\text { If } A_{j} \text { returns, } \\
\forall A \in \mathcal{G}_{k} \text { proceed to a final destination } x_{r}\end{array}$ \\
\hline$\overline{\mathcal{M}}_{3}:$ detour-together & $\begin{array}{l}A_{j} \text { leads } \mathcal{G}_{k} \text { to a goal } x_{p} \\
\text { All } A_{j} \in \mathcal{G}_{k} \text { follow } A_{i} \\
\text { If the goal is accomplished, } \\
\forall A \in \mathcal{G}_{k} \text { proceed to a final destination } x_{r}\end{array}$ \\
\hline
\end{tabular}

Because our interest is on generating cohesive group movements in a crowd simulation, we implement macro-plans only for the most common navigation strategies. We support three macro-plans, 'divide-and-proceed', 'divide-and-wait', and 'detour-together' as listed in Table 4. $A_{i}$ is a member of $\mathcal{G}_{k}$ and initiator of the macro-plan, and $A_{j} ;\left(A_{j} \in \mathcal{G}_{k}, \forall j \neq i\right)$ is a respondent actor. Suppose the 'divide-and-proceed' plan is chosen. $A_{i}$ leaves its group and proceeds to its own goal $x_{p}$ while $A_{j}$ keeps moving towards the group's original destination $x_{r}$. In the selection of the 'divide-and-wait' strategy, $A_{i}$ heads for its own goal $x_{p}$ by itself while the rest of group members stay at the current location. Once it achieves 
the goal, $A_{i}$ returns to where it left the group members. When all of the group members reunite, they resume the original navigation. Upon the selection of the 'detour-together' macro-behavior, $A_{i}$ leads all the members together to a goal $x_{p}$. When the goal is accomplished, they resume the original navigation from the goal location. In essence, during the detour, the group is in a 'follow-the-leader' macro-plan with $A_{i}$ in the role of the leader.

The selection of a macro-plan results in a set of desired position, velocity, and orientation values for those characters involved in a joint navigation. Table 5 shows an example of the macro-behavior outputs for $A$ and $B$ in our shopping mall scenario. $\mathcal{M}_{2}$ is selected after the coordination in Table 3 , and actors start executing the macro-behaviors at time $t_{8}$. We empirically set an appropriate constant time as a duration of visit in a shop.

Table 5. An example of macro-behavior action script for $A$ and $B, \mathcal{M}_{2}$ selection

\begin{tabular}{c|l|l|l|l}
\hline \multirow{2}{*}{ Macro-plan } & \multicolumn{4}{|c}{ Macro-behavior and time stamp } \\
\cline { 2 - 4 } & \multicolumn{1}{|c}{$A$, motion and activation time } & $B$, motion and activation time \\
\hline 1 & Walk to $x_{s}$ & $t_{8}: t_{7}+f_{\text {nod }}^{A}$ & Wait (Idle) & $t_{8}: t_{7}+f_{\text {nod }}^{A}$ \\
2 & Stay at $x_{s}$ & $t_{9}: t_{8}+f_{\text {walk_to_x }}^{A}$ & \\
3 & Walk to $x_{b}$ & $t_{10}: t_{9}+f_{\text {stay }}^{A}$ & & \\
4 & Walk & $t_{11}: t_{10}+f_{\text {walk_to_x }}^{A}$ & Walk & $t_{11}: t_{10}+f_{\text {walk_to_x }}^{A}$ \\
\hline
\end{tabular}

\section{Implementation and Results}

The system generates low-level collision-free motion steering decisions using the velocity obstacle model [1,8], employing a publicly available RVO2 Library [2]. We employed OpenGL-based rendering to visualize the animated characters driven by our simulation engine. Our crowd framework is tested on a desktop equipped with an Intel Core i7 $2.67 \mathrm{GHz}$ processor, $12 \mathrm{~GB}$ of RAM, and a Nvidia Quadro 5000 graphics card with 2.5GB device memory.

\subsection{Experiments}

We demonstrate the impact of the CG model on character behaviors and crowd simulations in this section. The simulation takes place in a generic shopping mall setting. Figure 3 gives an overview of our shopping mall model. The virtual shopping mall contains 6 restrooms and 82 stores as potential goals. We populated the shopping mall with 1000 characters, of which 800 are arranged into 200 social groups (e.g., family and friends), and the remaining 200 characters are individual actors in the crowd. A number of members in a group ranges from 2 to 6 , and is arbitrarily selected for each group. 


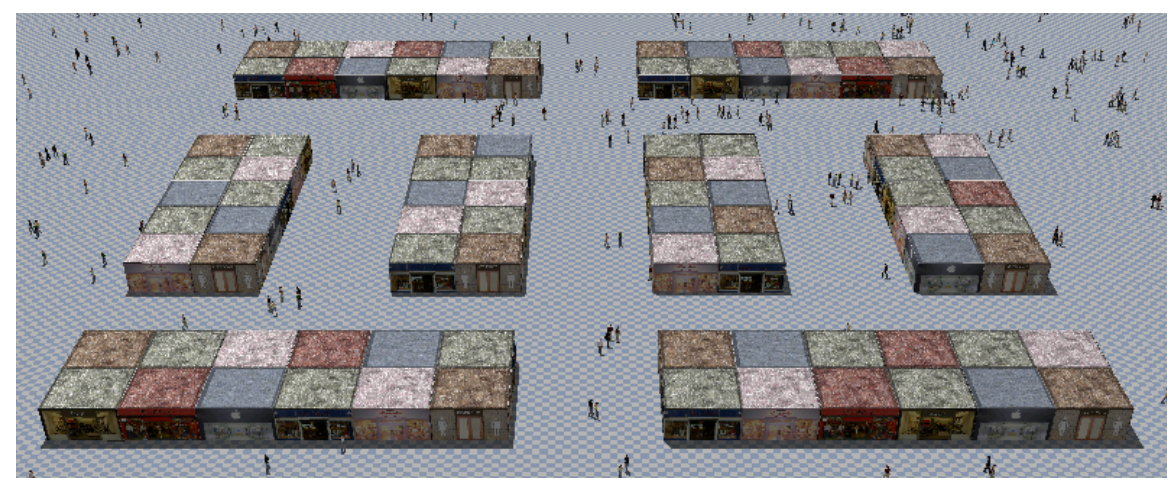

Fig. 3. A snapshot of the shopping mall model

\subsection{Effects in Character Behaviors}

Figure 4 shows characters communicating with other group members and presenting gestures. Depending on the spatial and environmental conditions, actors select different kinds of micro-behavior. Figure 4(a) shows a female character (labeled A) calling out to a friend to initiate communication. A man character (labeled B) is waving his hand to bring a colleague's attention in Figure 4(b). On the left side of him, a group of people (labeled C) is having a conversation.

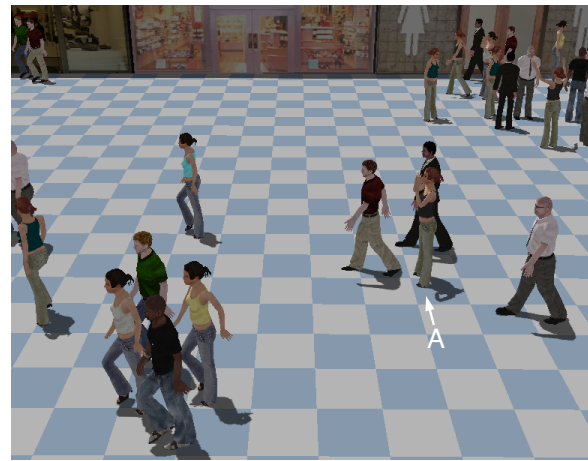

(a)

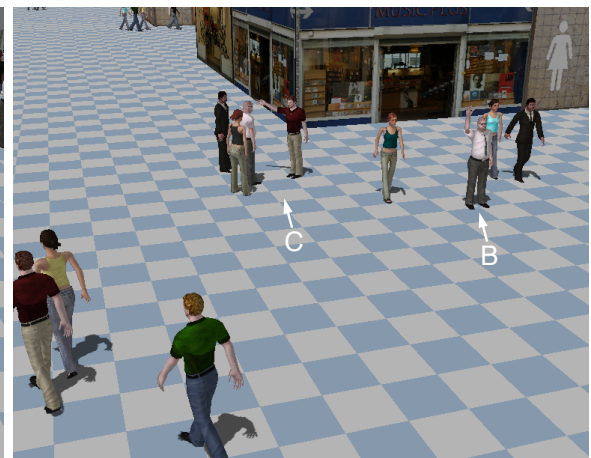

(b)

Fig. 4. (a) A female character calling out to a friend (b) A male character waving hand

Figure 5(a) shows several individuals (D, E, and F) and a couple (G) standing near a restroom, and waiting for their friends or family members. Individual characters are in idle poses. The couple is talking to each other, and the female character is presenting a hand gesture to the male character. In Figure 5(b), it is observed that a clustered movement emerges, because people in a group walk together and maintain a cohesive movement. 


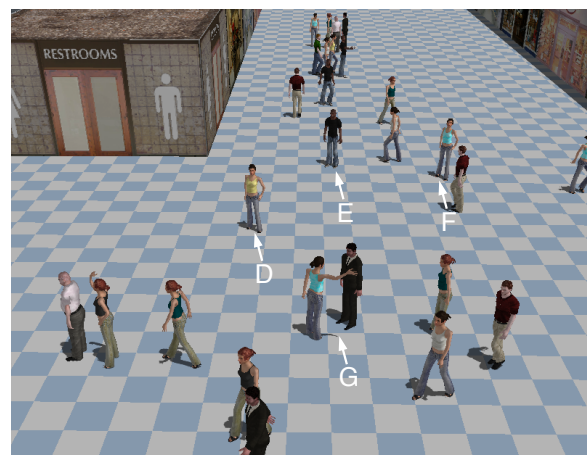

(a)

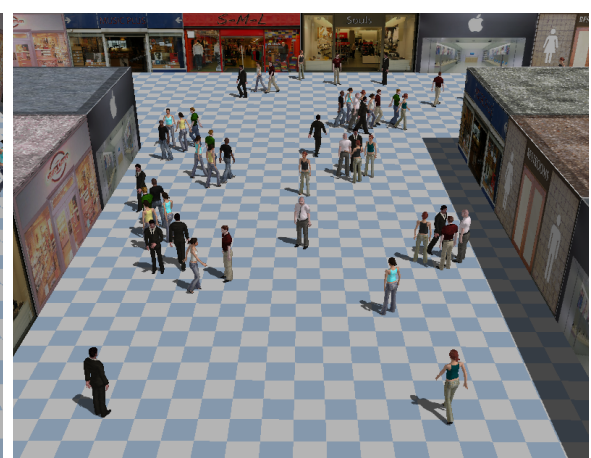

(b)

Fig. 5. (a) Individuals and a couple waiting for their members near a restroom (b) Group based movement

\section{Conclusion and Future Work}

We presented a crowd simulation framework that focuses on producing socially plausible animation behaviors of characters. Our high-level behavioral model for interaction and coordination is informed by Clark's well-researched common ground theory and hence inherits the social realism provided by the CG model. A character's motion is determined by considering temporal and spatial factors of the situation in which the character interacts with its group members. Therefore a sequence of character behaviors is not merely a chain of reactive gestures, but form socially meaningful interactions.

There are several possible improvements for our crowd modeling framework. On a presentation side, the actions of the characters were repetitive and not always smooth enough because of the lack of variety in motions in the database. We plan to add a richer set of communicative gestures and movements for microbehaviors (e.g., 'looking at' by head movement) that characters can display.

We will continue to improve the rendering engine for achieving good connectivity and smooth transitions in motions using motion synthesis techniques. Visualizing a large number of animated characters is a heavy computational process. To increase the rendering efficiency, we plan to employ the dynamic level-of-detail approach [26] to reduce the workload for the stages of graphics pipeline.

The two-level approach consisting of micro- and macro-behaviors allows flexible adaptation of our crowd model to different animation contexts. Since the set of human communicative actions remains consistent across application domains, the model of micro-behaviors is reusable. We want to apply our crowd simulation framework to different animation contexts by providing a pertinent repertoire of macro-behaviors to a target application, for example, a military coordination training scenario. 
We measured the arrival rate at a designated destination and congestion levels at key points to show the influence of the coordination model in the crowd simulations in $[22,23]$. However, the realism of crowd behaviors and dynamics cannot be fully confirmed with quantitative measures only. Therefore, we plan to conduct user perceptual evaluations to validate the simulation results.

\section{References}

1. van den Berg, J., Lin, M., Manocha, D.: Reciprocal velocity obstacles for real-time multi-agent navigation. In: Proc. of Robotics and Automation, 2008. ICRA 2008. IEEE Intl. Conf. on. pp. 1928 -1935 (May 2008)

2. van den Berg, J., Guy, S.J., Snape, J., Lin, M.C., Manocha, D.: RVO2 library: Reciprocal collision avoidance for real-time multi-agent simulation. http://gamma. cs.unc.edu/RVO2/ (2011)

3. Brogan, D.C., Johnson, N.L.: Realistic human walking paths. In: Proc. of the 16th Intl. Conf. on Computer Animation and Social Agents (CASA 2003). pp. 94-. CASA '03, IEEE Computer Society (2003)

4. Cassell, J., Vilhjálmsson, H.H., Bickmore, T.: Beat: the behavior expression animation toolkit. In: Proc. of the 28th annual conf. on Computer graphics and interactive techniques. pp. 477-486. SIGGRAPH '01, ACM (2001)

5. Clark, H.H., Brennan, S.: Grounding in communication pp. 127-149 (1991)

6. Coleman, J.S., James, J.: The equilibrium size distribution of freely-forming groups. Sociometry 24(1), pp. 36-45 (1961)

7. Endrass, B., André, E., Rehm, M., Lipi, A.A., Nakano, Y.: Culture-related differences in aspects of behavior for virtual characters across germany and japan. In: Proc. of the 10th Intl. Conf. on Autonomous Agents and Multiagent Systems - vol. 2. pp. 441-448. AAMAS '11 (2011)

8. Fiorini, P., Shillert, Z.: Motion planning in dynamic environments using velocity obstacles. International Journal of Robotics Research 17, 760-772 (1998)

9. Helbing, D., Moln, P., Farkas, I.J., Bolay, K.: Self-organizing pedestrian movement. Environment and Planning B: Planning and Design 28(3), 361-383 (2001)

10. Hoogendoorn, M., Soumokil, J.: Evaluation of virtual agents utilizing theory of mind in a real time action game. In: Proc. of the 9th Intl. Conf. on Autonomous Agents and Multiagent Systems: vol. 1. pp. 59-66. AAMAS '10 (2010)

11. Hughes, R.L.: A continuum theory for the flow of pedestrians. Transportation Research Part B: Methodological 36(6), 507 - 535 (2002)

12. James, J.: The Distribution of Free-Forming Small Group Size. American Sociological Review 18(5), 569-570 (1953)

13. Jan, D., Herrera, D., Martinovski, B., Novick, D., Traum, D.: A computational model of culture-specific conversational behavior. In: Proc. of the 7 th intl. conf. on Intelligent Virtual Agents. pp. 45-56. IVA '07 (2007)

14. Kapadia, M., Singh, S., Hewlett, W., Faloutsos, P.: Egocentric affordance fields in pedestrian steering. In: Proc. of the 2009 symposium on Interactive 3D graphics and games. pp. 215-223. I3D '09, ACM (2009)

15. Lau, M., Kuffner, J.J.: Behavior planning for character animation. In: Proc. of the 2005 ACM SIGGRAPH/Eurographics symposium on Computer animation. pp. 271-280. SCA '05, ACM (2005)

16. Lemercier, S., Jelic, A., Kulpa, R., Hua, J., Fehrenbach, J., Degond, P., AppertRolland, C., Donikian, S., Pettré, J.: Realistic following behaviors for crowd simulation. Comp. Graph. Forum 31(2pt2), 489-498 (May 2012) 
17. Li, Y., Wang, T., Shum, H.Y.: Motion texture: a two-level statistical model for character motion synthesis. ACM Trans. Graph. 21(3), 465-472 (Jul 2002)

18. Liu, C.K., Hertzmann, A., Popović, Z.: Composition of complex optimal multicharacter motions. In: Proc. of the 2006 ACM SIGGRAPH/Eurographics symposium on Computer animation. pp. 215-222. SCA '06 (2006)

19. Moussaïd, M., Perozo, N., Garnier, S., Helbing, D., Theraulaz, G.: The Walking Behaviour of Pedestrian Social Groups and Its Impact on Crowd Dynamics. PLoS ONE 5(4), e10047+ (Apr 2010)

20. Musse, S.R., Thalmann, D.: A Model of Human Crowd Behavior: Group InterRelationship and Collision Detection Analysis. In: Workshop Computer Animation and Simulation of Eurographics. pp. 39-52 (1997)

21. Narain, R., Golas, A., Curtis, S., Lin, M.C.: Aggregate dynamics for dense crowd simulation. ACM Trans. Graph. 28, 122:1-122:8 (December 2009)

22. Park, S.I., Quek, F., Cao, Y.: Modeling agent social joint actions via micro and macro coordination strategies. To appear in: Proc. of 2012 IEEE/WIC/ACM Intl. Conf. on Web Intelligence and Intelligent Agent Technology. WI-IAT '12 (2012)

23. Park, S.I., Quek, F., Cao, Y.: Modeling social groups in crowds using common ground theory. To appear in: Proc. of 2012 Winter Simulation Conference. WSC '12 (2012)

24. Pelachaud, C.: Multimodal expressive embodied conversational agents. In: Proc. of the 13th annual ACM intl. conf. on Multimedia. pp. 683-689. MULTIMEDIA '05 (2005)

25. Pelechano, N.: Crowd simulation incorporating agent psychological models, roles and communication. In: Proc. of the First Intl. Workshop on Crowd Simulation. pp. 21-30 (2005)

26. Peng, C., Park, S.I., Cao, Y., Tian, J.: A real-time system for crowd rendering: parallel lod and texture-preserving approach on gpu. In: Proc. of the 4th intl. conf. on Motion in Games. pp. 27-38. MIG '11 (2011)

27. Pettré, J., Laumond, J.P., Siméon, T.: A 2-stages locomotion planner for digital actors. In: Proc. of the 2003 ACM SIGGRAPH/Eurographics symposium on Computer animation. pp. 258-264. SCA '03 (2003)

28. Reynolds, C.W.: Flocks, herds and schools: A distributed behavioral model. SIGGRAPH Comput. Graph. 21, 25-34 (August 1987)

29. Shao, W., Terzopoulos, D.: Autonomous pedestrians. Graphical Models 69(5-6), 246 - 274 (2007), special Issue on SCA 2005

30. Stone, M., DeCarlo, D., Oh, I., Rodriguez, C., Stere, A., Lees, A., Bregler, C.: Speaking with hands: creating animated conversational characters from recordings of human performance. ACM Trans. Graph. 23(3), 506-513 (Aug 2004)

31. Thomas, G., Donikian, S.: Virtual humans animation in informed urban environments. In: Proc. of the Computer Animation. pp. 112-. CA '00, IEEE Computer Society (2000)

32. Tsai, J., Fridman, N., Bowring, E., Brown, M., Epstein, S., Kaminka, G., Marsella, S., Ogden, A., Rika, I., Sheel, A., Taylor, M.E., Wang, X., Zilka, A., Tambe, M.: Escapes: evacuation simulation with children, authorities, parents, emotions, and social comparison. In: Proc. of the 10th Intl. Conf. on Autonomous Agents and Multiagent Systems - vol. 2. pp. 457-464. AAMAS '11 (2011)

33. Wampler, K., Andersen, E., Herbst, E., Lee, Y., Popović, Z.: Character animation in two-player adversarial games. ACM Trans. Graph. 29(3), 26:1-26:13 (Jul 2010)

34. Yu, Q., Terzopoulos, D.: A decision network framework for the behavioral animation of virtual humans. In: Proc. of the 2007 ACM SIGGRAPH/Eurographics symposium on Computer animation. pp. 119-128. SCA '07 (2007) 